\title{
Nuevos avances en el conocimiento del síndrome postrombótico
}

\author{
F. GABRIEL BOTELLA, M. LABIÓS GÓMEZ, O. PORTOLÉS REPARAZ1, \\ J. CABANES VILA ${ }^{2}$
}

Servicio de Medicina Interna. Hospital Clínico Universitario de Valencia. ${ }^{1}$ Unidad de Epidemiología Genética y Molecular. Departamento de Medicina Preventiva. ${ }^{2}$ Departamento de Anatomía. Facultad de Medicina. Universidad de Valencia.

NEW ADVANCES IN THE UNDERSTANDING OF POST-THROMBOTIC SYNDROME

\section{RESUMEN}

La verdadera incidencia del síndrome postrombótico (SPT) no se conoce con precisión, aunque de la mayor parte de los estudios disponibles, parece deducirse que puede establecerse un año después de la trombosis venosa profunda (TVP) aguda de los miembros inferiores en el $17 \%$ al $50 \%$ de los pacientes. Inseparablemente unido a la hipertensión venosa que sigue al desarrollo de la incompetencia valvular, se acompaña de una serie de reacciones inflamatorias que incluyen el aumento de la permeabilidad endotelial, la unión de los leucocitos circulantes al endotelio, la infiltración por monocitos, linfocitos y mastocitos del tejido conectivo, y el desarrollo de infiltrados tisulares fibróticos y distintos marcadores moleculares. Al contrario que en las TVP, sabemos muy poco acerca de los factores que incrementan el riesgo de padecer un SPT, ya que el único identificado hasta ahora es la TVP recurrente. Actualmente disponemos de distintas escalas estandarizadas para su diagnóstico clínico, aunque el eco-Doppler duplex color es, actualmente, la técnica no invasiva de elección para detectar, localizar y evaluar la incapacidad venosa valvular y la obstrucción venosa crónica. Las modernas técnicas de imagen: tomografia computarizada (TC), resonancia magnética (RM) e isotópicas tienen un futuro prometedor, si bien se encuentran en fase de validación. Los fármacos flebotrópicos son la estrategia terapéutica de elección para los pacientes con SPT en los que no está indicada la cirugía o en los que ésta es un coadyuvante del tratamiento médico. Finalmente, la cirugía venosa profunda debe reservarse para todos aquellos pacientes que sufren de insuficiencia venosa crónica grave, con reflujo venoso significativo e hipertensión venosa ambulatoria.

PALABRAS CLAVE: Síndrome postrombótico. Eco-doppler.
ABSTRACT

The real incidence of the post-thrombotic syndrome (PTS) is not known precisely, though of the most part of the vailable studies, seems be deduced that it can be established a year after the deep venous thrombosis (DVT) acute of the inferior members in $17 \%$ to the $50 \%$ of the patients. Inseparably united to the venous hypertension that continues to the development of the incompetence valvular, is accompanied of a series of inflammatory reactions that include the increase in the permeability endothelial, the union of the circulating leukocytes at endothelium, the infiltration by monocytes, Iymphocytes and mastocytes of the connective tissue, and the development of infiltrated tissular fibrotics and different molecular markers. To the contrary that in the DVT, we know very little about the factors that increase the risk of suffering a PTS, since the only one identified up until now it is the recurrent DVT. Currently we have different scales standardized for their your clinical diagnosis, though the Echo-Doppler is, currently, the technique not invasive of election to detect, locate and evaluate the venous disability valvular and the venous obstruction chronicle. The modern technical of image: computed tomography $(C T)$, magnetic resonance $(M R)$ and isotopics have a prornising future, even though are found in validation phase. The phlebothropics drugs are the therapeutic election strategy for the patients with PTS in those which is not indicated the surgery or in those which this is a assisting of the medical treatment. Finally, the deep venous surgery must be reserved for all those patients that suffer from venous insufficiency serious chronicle, with meaningful venous reflux and ambulatory venous hypertension.

KEY WORDS: Post-thrombotic syndrome. Echo-doppler.

Gabriel Botella F, Labiós Gómez M, Portolés Reparaz O, Cabanes Vila J. Nuevos avances en el conocimiento del síndrome postrombótico. An Med Interna (Madrid) 2003; 20: 483-492.

\section{INTRODUCCIÓN}

La trombosis venosa profunda (TVP) de los miembros inferiores es una grave enfermedad con una incidencia aproximada de un caso/1.000 habitantes/año (1-3). Su curso clínico puede complicarse por la aparición de tasas significativas de embolias pulmonares (EP), hemorragias, TVP recurrentes y el desarrollo de graves secuelas postrombóticas $(4,5)$. Resulta, por tanto, sorprendente que la mayor parte de los numerosos estudios realiza- dos en los últimos quince años, se hayan centrado sobre sus complicaciones a corto plazo, mientras sus consecuencias tardías, pero no menos graves, como el síndrome postrombótico (SPT), hayan recibido tan poca atención en la literatura (6). Pero todavía es más sorprendente, si cabe, que, actualmente, su único factor de riesgo identificado sea la TVP recurrente ipsilateral (4). Su resultado lógico ha sido que, hasta el presente, el médico no pueda proporcionar a los pacientes con TVP una información individualizada sobre el pronóstico de su enfermedad (6).

Trabajo aceptado: 28 de abril de 2003 
Aunque estudios prospectivos recientes han demostrado que este síndrome caracterizado por edema, dolor, ectasias venosas y piel indurada, se manifiesta en el $17 \%$ al $50 \%$ de los enfermos dentro del primer año después del episodio trombótico agudo (6), y que el 30\% de este tipo de pacientes desarrollarán manifestaciones postrombóticas (4), su verdadera incidencia no ha sido establecida con precisión. Una posible explicación para esta amplia variabilidad sería que la mayor parte de las investigaciones realizadas hasta ahora se han limitado a series pequeñas o retrospectivas, siendo muy escasas las realizadas a largo plazo $(7,8)$. También a las grandes diferencias existentes entre ellas en cuanto a su diseño, definición del SPT, períodos de seguimiento efectuados y al elevado potencial de sesgos introducidos, al no diferenciar claramente las secuelas postrombóticas de las TVP recurrentes (9). Por todo ello es necesario la realización de nuevas investigaciones que nos permitan conocer con precisión el intervalo de tiempo transcurrido entre la TVP y la aparición del SPT (10), así como el desarrollo de métodos diagnósticos validados para evaluar las consecuencias de este último a largo plazo (6). Por otra parte, la verdadera incidencia del SPT en diferentes subpoblaciones de pacientes con TVP no ha sido adecuadamente establecida. No disponemos de datos, todavía, sobre predictores potenciales del SPT como la situación socioeconómica, factores ergonómicos,- porcentaje de tiempo empleado para ponerse de pie -, tipo y frecuencia de ejercicio, gravedad de los signos y síntomas de su presentación, comorbilidad -arteriopatía, insuficiencia cardiaca congestiva-, tiempo para alcanzar una anticoagulación adecuada durante su presentación inicial, uso de las heparinas de bajo peso molecular (HBPM), presencia de mutaciones genéticas asociadas con las trombofilias y modelos adecuados para la utilización de medias de compresión graduadas o de la compresión neumática intermitente $(6,11,12)$. Con respecto a la calidad de vida de este tipo de pacientes, es necesario responder a varias e importantes cuestiones: ¿después de una TVP hay diferencias entre los pacientes con y sin SPT? ¿El SPT varía con la duración del tratamiento, origen y extensión de la TVP? ¿Es comparable la calidad de vida con la de otras enfermedades crónicas? (6)

Para responder a todos estos interrogantes es necesario realizar amplios estudios prospectivos en enfermos con TVP que nos proporcionen una información exhaustiva basada en la evidencia científica, respecto a su pronóstico a largo plazo. Ello nos ayudará a cuantificar la prevalencia y los costes socioeconómicos del SPT, por una parte, y a identificar sus factores de mal pronóstico, por otra. En definitiva, a desarrollar nuevas estrategias preventivas en todos aquellos pacientes con riesgo de desarrollar esta complicación $(6,12)$.

\section{MAGNITUD DEL PROBLEMA}

La verdadera incidencia del SPT no se conoce con precisión. En la mayor parte de los estudios realizados en los últimos 60 años varía entre el 20 y el 100\% (13-15). Una posible explicación para esta gran variación se debería al limitado número de investigaciones que miden prospectivamente la incidencia del SPT, y a que no son comparables debido a las diferencias en el tiempo de seguimiento, selección de los pacientes y definición del síndrome $(6,9,15)$. Stradness et al (16), en un estudio realizado en 61 pacientes con TVP encuentran que, 39 meses después, el 67\% tenían dolor o edema en el miembro afecto. Beyth et al (17), en otro estudio realizado en 124 pacientes con TVP, demuestran que a los 8 años de seguimiento, el $42 \%$ presentaban por lo menos un síntoma sugestivo de SPT, aunque no se realizó una exploración física para confirmar su diagnóstico.

Otros dos estudios han demostrado que el SPT en la mayor parte de los casos se establece entre el primer y segundo año después de la TVP. Prandoni et al (4), en un estudio prospectivo de 355 pacientes con un primer episodio trombótico y un seguimiento bianual durante más de ocho años, demuestran que 84 pacientes $(23,7 \%)$, desarrollaron un SPT, de los que el $30,2 \%$ tenían manifestaciones graves. La incidencia acumulativa fue del 17,3 y del $22,8 \%$ al primer y segundo año de seguimiento, respectivamente, y se incrementa gradualmente hasta el $29,1 \%$ a los ocho años. Lo que contrasta con las cifras obtenidas en otros estudios previos y que podría explicarse, en parte, por cuatro factores. Primero, el $15 \%$ de los pacientes sólo tenían una TVP poplítea o de la pantorrilla, lo que comparado con las trombosis proximales, puede asociarse con un bajo riesgo de padecer un SPT. Segundo, las medias de compresión, que parecen reducir la incidencia del SPT, fueron empleadas sistemáticamente. Tercero, se utilizaron criterios diagnósticos estandarizados con lo que se evitaron todos aquellos signos y síntomas no específicos que en anteriores estudios podrían haberse etiquetados como típicos de un SPT. Finalmente, determinados factores específicos pueden haber contribuido a su baja incidencia (su origen, el que todos los pacientes fueran ambulatorios y que ninguno tenía TVP recurrentes). Un estudio aleatorio (18) para evaluar los beneficios de las medias de compresión (40 mmHg de presión en el tobillo), realizado en 194 pacientes con una TVP proximal previa, y en el que se utilizó el sistema de puntuación diagnóstico de Villalta et al (19), demostró que el SPT de tipo medio aparecía dentro de los 12 meses siguientes en el $25 \%$ de los pacientes tratados con la media de compresión, en comparación con el $50 \%$ del grupo control. Para el SPT grave, estas cifras fueron del 10 y del $20 \%$, respectivamente. Un estudio reciente (11) ha demostrado un incremento estadístico y clínicamente significativo de la incidencia del SPT $(30 / 110=27,3 \%$; IC 95\%, $18,9-35,6 \%)$, un año después de padecer una TVP proximal sintomática.

Por lo tanto, de la mayor parte de los estudios disponibles, parece deducirse que el SPT se establece un año después de la TVP aguda en el $17 \%$ al $50 \%$ de los pacientes. A pesar de la creencia generalizada de que son necesarios de cinco a diez años para que se manifieste el SPT, estos tres estudios $(4,11,18)$ demuestran que la mayor parte de los casos se vuelven clínicamente aparentes entre el primer y el segundo año después del episodio trombótico agudo.

\section{REPERCUSIÓN SOCIOECONÓMICA DEL SPT}

En cualquier caso, sus consecuencias pueden adquirir caracteres de extraordinaria importancia. En España, se calcula que el $1,5 \%$ de la población total y el $11,3 \%$ de los pacientes con patologías venosas padecen complicaciones postrombóticas. Además, el $2,5 \%$ de las bajas laborales por enfermedad se deben a procesos trombóticos venosos, lo que supone unos gastos superiores a los de todas las formas de arteriosclerosis juntas, incluidos el infarto de miocardo y las 
isquemias periféricas (20). El coste económico en nuestro país de un paciente con TVP aguda era ya en 1992 de 462.550 ptas. con tratamiento anticoagulante; 771.649 ptas con interrupción de la vena cava inferior; 963.630 ptas con trombectomía venosa y 2.504 .350 ptas. con fibrinolíticos (21). Todo ello sin considerar los gastos acumulativos que suponía el tratamiento tópico o quirúrgico paliativo del SPT en sus diferentes estadios. En el Reino Unido el coste anual del SPT se calcula en 400.000.000 de £. Asimismo, los países de la Union Europea dedican del $1,5 \%$ al $2 \%$ de sus gastos sanitarios al tratamiento de las enfermedades venosas crónicas (22).

\section{FISIOPATOLOGÍA Y DETERMINANTES DEL DESARROLLO DEL SPT}

El SPT es el resultado de la hipertensión venosa que sigue al desarrollo de la incompetencia valvular, al reflujo, y/o a la obstrucción venosa, con una función linfática o microcirculatoria anormales $(10,23)$. La incompetencia valvular es la consecuencia del daño sufrido por las válvulas venosas en el momento del episodio trombótico agudo, o durante la fase posterior de recanalización venosa. Si ésta es incompleta aparece una oclusión del flujo venoso, lo que lleva al desarrollo de la circulación colateral por medio de las venas superficiales y perforantes, que gradualmente pueden volverse incompetentes y varicosas debido a su progresiva dilatación (24).

\section{LAS CÉLULAS ENDOTELIALES MICROVASCULARES}

La hipertensión venosa, secuela final de todo este proceso, se transmite directamente hacia la circulación superficial, afectando principalmente a las células endoteliales, que tienen un papel muy importante en las reacciones inflamatorias. Cambios sutiles en el revestimiento de su glicocalix y en las fisuras de sus uniones interendoteliales pueden estar implicados en el edema del SPT. La pérdida de proteínas plasmáticas puede llevar a la coagulación del fibrinógeno en el espacio extravascular y a la formación de acúmulos periarteriolares de fibrina (25).

Es posible que las células endoteliales microvasculares de los pacientes con SPT sean metabólicamente activas y presenten un contenido ribosómico prominente y un núcleo eucromático (26). Existen evidencias que sugieren una activación de la síntesis proteíca al menos en algunas regiones de las venas afectadas. Especímenes de úlceras venosas de los miembros inferiores causadas por el SPT muestran sobre el endotelio un incremento de la expresión de las moléculas de adhesión intercelular de clase 1 (ICAM-1) y de las moléculas de adhesión de las células vasculares de clase 1 (VCAM-1), pero menos expresión de selectina E, en comparación con la piel sana (27).

¿Qué causas pueden desencadenar la inducción de la síntesis proteíca? Se han señalado varias posibilidades para explicarla. Una de ellas es que puede ser debida a una estimulación hormonal, por ejemplo, durante la producción precoz y mantenida de progesterona al comienzo del embarazo. La hipoxia por sí misma, puede inducir el factor-1 inducible por hipoxia (HIF-1) como un factor de transcripción compuesto de subunidades alfa y beta HIF-1 (28). El HIF-1 transactiva múltiples genes, incluyendo el factor de crecimiento vasculoendotelial (VEGF), que tiene un papel fundamental en la homeostasis del oxígeno. El HIF-1también desempeña un papel importante en el control de la regulación del VEFG en la isquemia retiniana (29), el cual a su vez puede ser detectado en el plasma de los pacientes afectados (30), aunque su papel en la enfermedad venosa no ha sido investigado.

Por otra parte, una presión hidrostática anormal en el SPT, probablemente, se acompañe de un cambio tanto en la distribución del flujo como en la tensión de cizallamiento de los fluídos sobre el endotelio (31). Todo lo cual apunta al hecho de que muchas de las funciones endoteliales sean controladas por ese tipo de tension. Por ejemplo, la tension de cizallamiento afecta directamente a la fluidez de la membrana (29) y a la fosforilización de la proteína G (30); modula la cascada de señalización (32), la producción de radicales libres de oxígeno, y afecta directamente a los distintos factores de transcripción nuclear (33).

\section{LEUCOCITOS CIRCULANTES}

La expresión de las moléculas de adhesión de las células endoteliales mantiene la entrada de leucocitos en las regiones microvasculares atrapándolos en los capilares y uniéndolos a las vénulas postcapilares (34). En cualquier órgano, este proceso es aumentado posteriormente si los leucocitos circulantes son activados con un incremento de la rigidez celular debido a la polimerización de la actina y a la formación de pseudópodos así como a la expresión de las moléculas de adhesión (35). La mayoría de las células observadas en los tejidos del SPT son neutrófilos, linfocitos T y monocitos $(33,34)$, acompañados por macrófagos y acúmulos de mastocitos (36). Como en el caso de las células endoteliales, la activación leucocitaria puede ser determinada por la tensión de cizallamiento de los fluidos (37). El aumento de los mediadores inflamatorios o la supresión del óxido nítrico (ON) a su vez suprime la respuesta a la tensión de cizallamiento y devuelve las células a un estado más proinflamatorio (38).

\section{CÉLULAS INTERSTICIALES}

En los pacientes con SPT hay un aumento de los mastocitos y los macrófagos en la vecindad de las arteriolas y de las vénulas postcapilares, que puede asociarse con la hipertensión venosa inducida por la extravasación de hematíes y la activación de las células endoteliales. Todo lo cual representa un potente estímulo tanto para el proceso inflamatorio como para la remodelación y reparación tisular posterior (39).

Las células mastocíticas humanas de clase 1 (HMC-1) se adhieren espontáneamente a la fibronectina, laminina, y colágeno tipo I y III, todos ellos componentes de los manguitos perivasculares (40). La cimasa, enzima de los mastocitos y potente activador de la matriz de la metaloproteinasa 1 y 3 $(39,40)$, causa la liberación del factor de crecimiento tumoral latente $\beta 1$ (TGF- $\beta 1$ ) secretado por las células endoteliales activadas, fibroblastos y plaquetas de las matrices extracelulares (41). Por otra parte, la liberación y activación del TGF-ß1 inicia una cascada de eventos en los que los macrófagos y los fibroblastos son reclutados para reparar las lesiones provocadas, y estimulados para producir fibroblastos mitógenos y proteínas del tejido conectivo, respectivamente (42). 


\section{EVIDENCIA MICROCIRCULATORIA DE LA INFLAMACIÓN EN LA HIPERTENSIÓN VENOSA}

La reperfusión subsiguiente al proceso isquémico provocado por la obstrucción venosa se acompaña de una elevación sistemática de la presión microvascular, lo que rápidamente lleva a una activación dependiente de selectina de los neutrófilos en el endotelio venular (23). Debido a la reperfusión, hay un aumento considerable de los leucocitos que se extienden sobre el endotelio venular y los tejidos adyacentes. Las células de los tejidos instersticiales están sujetas a apoptosis y los segmentos superiores del vaso ocluido están sometidos a hemorragias dentro del espacio intersticial. Los sitios preferenciales para la diátesis de los hematíes, que ya tiene lugar durante el período oclusivo inicial, son las vénulas postcapilares más pequeñas (43).

Una parte significativa del tejido dañado la origina la liberación de los radicales libres de oxígeno generados por las xantinaoxidasas y la inhibición de la producción del ON seguido por la unión de la membrana endotelial y los leucocitos así como el aumento de la muerte celular parenquimatosa $(42,44)$. Ésta ocurre con o sin leucocitos, aunque la deplección de estos últimos en los modelos experimentales proporciona algún grado de protección de la función de los órganos parenquimatosos (45). El proceso se acentúa en presencia de otros factores de riesgo cardiovascular, como la hipercolesterolemia (46). La hipertensión venosa central lleva también a la infiltración leucocitaria de la piel (47).

\section{MECANISMOS DESENCADENANTES DE LA INFLAMACIÓN EN EL SPT}

Una de las contribuciones más importantes a la elevación de la presión venosa es la pérdida de la capacidad de las válvulas venosas para cerrarse completamente y prevenir el reflujo sanguíneo (23). Hay dos mecanismos físicos para explicar el fallo venoso valvular. Una o ambas de las lengüetas se acortan, se desprenden de la pared venosa, e incluso pueden perforarse. Un mecanismo alternativo puede ser una simple distensión venosa de tal modo que incluso una lengüeta normal no tenga la longitud suficiente para cerrarse adecuadamente (23). La simple distensión del anillo valvular puede ser suficiente para causar un reflujo venoso. Tal distensión se debe al reblandecimiento de la pared, probablemente, bajo la influencia hormonal (progesterona) (48), o a la activación crónica en el plasma (34), o a la pérdida de resistencia en la pared venosa y al soporte insuficiente para las estructuras tisulares circundantes (23).

Además del estrés de los fluidos y los mecanismos moleculares, es posible que otros factores, como las infecciones bacterianas o fúngicas, puedan servir como cofactores que desencadenen o amplifiquen el proceso inflamatorio en estos pacientes. Las infecciones bacterianas de distintas fuentes (pulmón, intestino) pueden expresarse sistemáticamente y servir para aumentar las reacciones inflamatorias en presencia de un estrés de la pared venosa o de una fuerza de corte anormal (23). Además, los factores ambientales, como el tabaco, regulan los leucocitos y/o el endotelio (49), mientras los componentes dietéticos -por ejemplo, la hipercolesterolemia-, son proinflamatorios. En modelos animales se ha demostrado que inducen una respuesta más vigorosa a la isquemia y a la reperfusión en relación a sus controles (46). Así mismo puede haber defectos genéticos que participen en el desarrollo de la enfermedad valvular independientemente de la hipertensión venosa (43).

\section{FACTORES DE RIESGO DEL SPT}

Al contrario que en las TVP, se sabe muy poco acerca de los factores que incrementan el riesgo de padecer un SPT (6). Parece haber una mínima correlación entre la gravedad de los hallazgos venográficos ( por ejemplo, el grado de oclusión ) del trombo inicial y el subsiguiente desarrollo del síndrome (50). Puede haber una relación entre este y la localización de la TVP $(15,51)$, aunque otras investigaciones no han demostrado esta asociación debido, posiblemente, a su falta de rigor metodológico (52). La terapia trombolítica de la TVP (administrada local o sistémicamente), a pesar de su eficacia en la lisis del trombo, no ha demostrado de forma consistente que pueda reducir el riesgo consiguiente de padecer un SPT $(53,54)$.

Prandoni et al (4), han demostrado que la TVP recurrente ipsilateral es un fuerte predictivo de SPT ( odds ratio 6,4 ). Últimamente (55), se ha apuntado la posibilidad de que los niveles de dímero $\mathrm{D}$ tienden a incrementarse en un número significativo de pacientes en el primer mes después de suprimir los anticoagulantes orales (AO) administrados tras una TVP. Este hecho fue más frecuente en aquellos pacientes con una trombosis idiopática o secundaria a factores de riego persistentes. Un dímero D normal, tres meses después de la supresión de los $\mathrm{AO}$, tiene un alto valor predictivo negativo para la TVP recurrente, mientras que la presencia de un dímero $\mathrm{D}$ aumentado se asocia con un riesgo significativamente elevado de padecer una TVP recurrente. Estos hallazgos sugieren que el dímero D puede tener un papel no sólo en el diagnóstico y seguimiento de la trombosis aguda sino también en el diseño de nuevas estrategias para optimizar la prevención de las TVP recurrentes y por ende del SPT (55).

Otros hallazgos clínicos como el aplazamiento de la visita al médico por parte del paciente, factores de riesgo trombótico, historia familiar y déficit de proteína $\mathrm{C}$ y S y antitrombina III, no han demostrado ser factores de riesgo del SPT (6). Tampoco se ha encontrado ninguna relación entre este síndrome y la mutación del factor $\mathrm{V}$ Leiden, resistencia a la proteína $\mathrm{C}$ activada, anticoagulante lúpico, hiperhomocisteinemia o factores desencadenantes de la TVP (embarazo, puerperio, aborto, anticonceptivos orales, cirugía, traumatismos, o inmovilización prolongada) (56), lo cual no es sorprendente, ya que el grado y el tipo de daño venoso es inducido por el trombo, independientemente del desencadenante trombótico. Dado que los pacientes trombofílicos tienen una alta incidencia de TVP recurrentes, podría esperarse que en los que las presentasen hubiera una asociación entre los defectos de coagulación y el SPT. Extremo este que no se ha podido demostrar debido a que como una vez diagnosticados reciben profilaxis cuando están expuestos a situaciones de riesgo, no muestran una recurrencia diferente a la de los no trombofílicos (56).

Largos períodos de anticoagulación oral podrían hacer disminuir el riesgo de SPT, en comparación con otros tratamientos de corta duración para reducir las TVP recurrentes, lo que puede ser particularmente cierto para las trombosis idiopáticas (6). Un ensayo clínico aleatorio (57) de pacientes con un primer episodio de tromboembolismo idiopático tuvo que ser 
interrumpido precozmente debido a que los que recibieron tres meses de tratamiemto anticoagulante presentaban unas tasas de recurrencia anuales del $27,4 \%$, en comparación con el $1,3 \%$ de los que fueron anticoagulados durante dos años. Por el contrario, las tasas anuales de hemorragias mayores por paciente/año fueron del 3,8\% en este último grupo, en comparación con el otro grupo en el que no hubo ninguna. Agnelli et al (58), en un estudio aleatorio en el que los pacientes con TVP idiopática fueron tratados tres meses o un año con AO y un seguimiento de al menos dos años, demuestran que el beneficio clínico asociado con la anticoagulación mantenida durante un año desaparece una vez esta es interrumpida. De todo ello se deduce que el tratamiento con $\mathrm{AO}$ reduce el riesgo de recurrencia durante su uso -reducción del riego relativo, aproximadamente en un 90\%-, aunque se asocia con una tasa anual de hemorragias mayores del 1-4\%.

Otros factores predictivos de SPT incluyen la edad, el sexo femenino, la terapia hormonal, las venas varicosas y la cirugía abdominal (6). Un estudio retrospectivo realizado en mujeres jóvenes con un seguimiento de un año después de la TVP, encuentra que un índice de masa corporal (IMC) superior a 22 $\mathrm{kg} / \mathrm{m}^{2}$ se asocia con un riesgo cinco veces mayor de desarrollar SPT (56). Sin embargo, hasta la fecha ningún estudio ha demostrado que la pérdida de peso mejore el síndrome (6).

\section{DIAGNÓSTICO DEL SPT.}

Los métodos actualmente disponibles para la valoración del diagnóstico del SPT incluyen: el diagnóstico clínico, las pruebas de evaluación y cuantificación macrocirculatorias (flebografía, eco-Doppler-duplex color y la angiorresonancia magnética [ARM]) y microcirculatorias (flujometría laser Doppler, medición transcutánea de la presión parcial de $\mathrm{O}_{2} \mathrm{y}$ $\mathrm{CO} 2$ y la pletismografía por impedancia)

\section{DIAGNÓSTICO CLÍNICO}

Los mecanismos fisiopatológicos que se suceden en un síndrome obstructivo venoso provocan cambios estructurales en los tegumentos que circundan el sector afectado, modificando su trofismo. Este es el motivo por el que aparecen: dolor en la bipedestación, edema, y alteraciones cutáneas (pigmentación, dermatitis de estasis, o hipodermitis) (59). El prurito y los cambios eczematosos de la piel son frecuentes y una proporción de pacientes desarrollarán venas prominentes y tortuosas, junto con otros síntomas más subjetivos, como pesadez y distensión cutánea (9). Las ulceraciones, a menudo precipitadas por un traumatismo, son característicamente crónicas e indoloras, con una alta tasa de recurrencia. Raramente, los pacientes con una obstrucción persistente pueden experimentar una claudicación venosa, que en algunos aspectos imita a la obstrucción arterial (59).

Su diagnóstico se basa esencialmente en el desarrollo de las manifestaciones clínicas, anteriormente mencionadas, en pacientes con una historia de TVP, independientemente de la presencia de alteraciones venosas demostradas por procedimientos invasivos o no. En ausencia de signos y síntomas, la demostración de estas alteraciones,- reflujo venoso, obstrucción venosa persistente, o ambas -, no es suficiente para poder diagnosticar a un paciente con historia de TVP previa de SPT (9).
Aunque el cuadro clínico del SPT es clásico (59), hay una gran variación entre los estudios publicados respecto a su clasificación clínica. Actualmente, disponemos de dos sistemas estandarizados de medición de la gravedad del síndrome. El primero (19), se basa únicamente en la utilización de una escala estandarizada de signos y síntomas clínicos (Tabla I). El segundo, también conocido como CEAP (60) (Clínica, Etiología, Anatomía, Fisiopatología), combina una serie de signos clínicos, con un sistema de modificadores que reflejan la causa subyacente (congénita, primaria, o secundaria a la TVP), la distribución anatómica (superficial, profunda, o de las venas perforantes), y las condiciones fisiopatológicas (reflujo, obstrucción, o ambos). Los síntomas no son considerados. Desde nuestro punto de vista no resulta operativo en la práctica clínica, ya que utiliza un complicado y sofisticado sistema de puntuación, además de no estar validado comprehensivamente (24) (Tabla II).

\section{TABLA I}

ESCALA ESTANDARIZADA PARA EL MANEJO CLÍNICO DEL SPT

\begin{tabular}{ll}
\hline Síntomas clínicos* & Signos clínicos* \\
\hline & Edema pretibial \\
Pesadez & Induración de la piel \\
Dolor & Hiperpigmentación \\
Calambres & Nuevas ectasias venosas \\
Prurito & Cianosis \\
Parestesias & Compresión dolorosa de la pantorrilla \\
& Úlceras cutáneas
\end{tabular}

Definición del SPT

Grave: úlcera en una ocasión o puntuación $\geq 15$ medida en dos visitas consecutivas

Moderado: entre 5 y 14 puntos en dos visitas consecutivas

Ausente: $\leq 4$ puntos

* A cada uno de los signos o síntomas se le asigna una puntuación entre 0 y 3

No se tomó en consideración la ausencia o la presencia de una úlcera en el miembro afecto. Tomado de Villalta et al (19).

\section{PRUEBAS INVASIVAS Y NO INVASIVAS}

La flebografía ascendente hasta hace pocos años era el método de elección para demostrar la elasticidad de las venas, definir su anatomía, ayudar a distinguir entre una enfermedad venosa primaria y secundaria (SPT) y detectar la incompetencia de las venas perforantes (12).

Los criterios flebográficos ascendentes actuales para el diagnóstico del SPT son la presencia de una oclusión total en algún punto del segmento venoso afectado -al igual que en la TVP-, o un estrechamiento superior al $50 \%$ de por lo menos la mitad del segmento en cuestión, al menos en dos proyecciones radiográficas diferentes (61). Otros criterios, como una falta de llenado de un segmento venoso o la interrupción abrupta de la columna de contraste en una sola proyección, un relleno intraluminal inconstante, y la falta de visión de una o más venas de la pantorrilla, son menos fiables y no deberían utilizarse para su confirmación diagnóstica. Aunque la flebografía ha sido considerada el estandar de oro en la detección del lugar y extensión de la obstrucción venosa crónica, no puede proporcionar un control funcional cuantitativo de su 
TABLA II

ESCALA CEAP PARA EL M ANEJO CLÍNICO DE LA AFECTACIÓN VENOSA EN EL SPT

\begin{tabular}{|c|c|c|c|c|}
\hline Atributo & Ausente $=0$ & M edio $=1$ & M oderado $=2$ & Grave $=3$ \\
\hline Dolor & No & $\begin{array}{l}0 \text { casional, no restringe } \\
\text { la actividad, ni requiere } \\
\text { analgésicos }\end{array}$ & $\begin{array}{l}\text { Diario, limitación } \\
\text { moderada de la actividad } \\
\text { Ocasionalmente } \\
\text { requiere analgésicos }\end{array}$ & $\begin{array}{l}\text { Diario, limitación } \\
\text { importante de la actividad o } \\
\text { requiere uso diario } \\
\text { de analgésicos }\end{array}$ \\
\hline Venas varicosas & No & Escasas & $\begin{array}{l}\text { Mútiples: confinadas } \\
\text { al muslo o pantorrila }\end{array}$ & Extensas \\
\hline Edema venoso & No & $\begin{array}{l}\text { Solo edema vespertino } \\
\text { del tobillo }\end{array}$ & $\begin{array}{l}\text { Edema nocturno por } \\
\text { encima del tobillo }\end{array}$ & $\begin{array}{l}\text { Edema nocturno por encima } \\
\text { del tobillo, que requiere } \\
\text { cambios en su actividad } \\
\text { y elevación de } \\
\text { los miembros inferiores }\end{array}$ \\
\hline Pigmentación cutánea & Ninguna o focal & Limitada a un área concreta & $\begin{array}{l}\text { Difusa, pero inferior a } 1 / 3 \\
\text { del total de la pantorrila } \\
\text { o pigmentación } \\
\text { de aparacion reciente }\end{array}$ & $\begin{array}{l}\text { Difusa, pero superior a } 1 / 3 \text { del } \\
\text { total de la pantorilla } \\
\text { y pigmentación de aparición } \\
\text { reciente }\end{array}$ \\
\hline Inflamación & No & $\begin{array}{l}\text { Celulitis media, limitada } \\
\text { al área marginal } \\
\text { periulcerosa }\end{array}$ & $\begin{array}{l}\text { Celulitis moderada, } \\
\text { inferior a } 1 / 3 \text { de la } \\
\text { pantorrila }\end{array}$ & $\begin{array}{l}\text { Celulitis grave, inferior } \\
\text { o superior a } 1 / 3 \text { de la } \\
\text { pantorrilla, o eczema venoso } \\
\text { significativo }\end{array}$ \\
\hline Induración & No & $\begin{array}{l}\text { Focal, circunmaleolar } \\
(<5 \mathrm{~cm})\end{array}$ & $\begin{array}{l}\text { M edia o lateral }<2 / 3 \text { del } \\
\text { total de la pierna }\end{array}$ & 2/3 o más del total de la pierna \\
\hline № de úlceras activas & Ninguna & Una & Dos & Más de tres \\
\hline $\begin{array}{l}\text { Duración de las úlceras } \\
\text { activas }\end{array}$ & No & M enos de tres meses & $\begin{array}{l}\text { Más de tres meses, } \\
\text { una úlcera }\end{array}$ & $\begin{array}{l}\text { Más de tres meses, una } \\
\text { úlcera }\end{array}$ \\
\hline $\begin{array}{l}\text { Tamaño de las } \\
\text { úlceras activas }\end{array}$ & No & $\begin{array}{l}\text { M enos de dos cm } \\
\text { de diámetro }\end{array}$ & $\begin{array}{l}\text { De dos a seis } \mathrm{cm} \\
\text { de diámetro }\end{array}$ & M ás de seis cm de diámetro \\
\hline Terapia compresiva & No & Utilización irregular & $\begin{array}{l}\text { Las utiliza la mayor parte } \\
\text { de los días }\end{array}$ & Las utiliza siempre \\
\hline
\end{tabular}

Tomado de Rutherford et al (60).

gravedad o de la suficiencia de las venas colaterales. En el 10$20 \%$ de los casos, la venografía no revela con la suficiente claridad algún segmento venoso. Además está contraindicada en pacientes con insuficiencia renal y puede presentar una serie de reacciones adversas que incluyen alergia al contraste, necrosis cutánea, dolor local y, hasta en el 3\% de los casos, tanto el agravamiento como la producción de una nueva trombosis (62).

Actualmente, la flebografía ascendente es necesaria únicamente en un pequeño número de pacientes que presentan anomalías anatómicas o malformaciones, o cuando la cirugía sobre el sistema venoso profundo esté indicada (63).

La flebografía descendente sirve para demostrar la existencia de reflujo tanto en el sistema venoso profundo como en el superficial, para determinar los puntos de filtración del contraste desde la pelvis a los miembros inferiores y desde el sistema venoso profundo al superficial. También puede utilizarse para tener información sobre la localización anatómica y la morfología de las válvulas venosas, examinar la extensión del reflujo, delinear la anatomía venosa en casos complejos y diferenciar una enfermedad venosa primaria de una secundaria (12). Además, es absolutamente necesaria en los pacientes que con una insuficiencia valvular venosa primaria vayan a ser sometidos a una valvuloplastia, o en aquellos que con una insuficiencia valvular venosa secundaria se les realice una sustitución valvular (63). Las críticas que se le hacen a esta exploración es que al igual que la flebografía ascendente, presenta las mismas complicaciones potenciales, y además es un método invasivo y caro, y no puede evaluar la capacidad valvular de las venas periféricas cuando hay unas válvulas competentes en la porción superior de la pierna, lo que provoca falsos negativos. Los falsos positivos son también posibles debido a la relativamente alta densidad del medio de contraste que puede extenderse hacia la parte inferior del miembro a través de las válvulas normales, pero parcialmente abiertas $(12,63)$.

Actualmente, el Eco-Doppler duplex color es la técnica no invasiva de elección para detectar, localizar y evaluar la incapacidad venosa valvular y la obstrucción venosa crónica. Una vena totalmente trombosada será incompresible y estará exenta de señales Doppler (64). Una vena parcialmente trombosada será escasamente compresible y las señales Doppler se obtendrán a partir de la superficie de la luz. Con el color, la trombosis total es inmediatamente identificada, dado que la ausencia del mismo será detectado en la vena identificada por efecto de la compresión. Con la oclusión parcial habrá una 
invasión de la imagen del flujo que no será lo suficientemente extensa como para rellenar enteramente la luz del vaso. El flujo en los canales colaterales es fácilmente localizable. Es menos probable que estos se confundan con la vena principal cuando se utiliza el registro del duplex o el del flujo-color, puesto que la localización de la vena se hace aparente a partir de las señales detectadas (64). La obstrucción venosa crónica puede diferenciarse de la aguda de varias formas. Mientras una vena agudamente trombosada tiende a distenderse más allá de un diámetro considerado generalmente normal, la venas crónicamente ocluidas a menudo están contraídas y tienen paredes gruesas que pueden ser más ecogénicas debido a la fibrosis, aunque pueden mostrar algo de flujo sanguíneo a través de las recanalizaciones venosas. El tiempo exacto de aparición de estos cambios es desconocido. Por ello, resulta arriesgado calificar un trombo de crónico basándose únicamente en criterios ecográficos. Ocasionalmente, existen calcificaciones y los trombos residuales también pueden ser más ecogénicos (64). El flujo retrógrado es señalado por un espectro invertido o por un cambio en el mapa de flujo-color que vira del azul al rojo. Las imágenes de las válvulas venosas a menudo se ven sobre una escala de grises. Los sinusoides valvulares normales tienen una configuración elíptica y las lengüetas son delgadas y móviles. Los sinusoides valvulares dañados están distorsionados, pueden contener material ecogénico y las lengüetas, cuando son visibles, son cortas y gruesas. Cuando éstas son adecuadamente visualizadas en tiempo real y su movimiento claramente discernido, la competencia valvular puede calcularse directamente. Las venas normales presentan un reflujo -tiempo de cierre valvular-, entre 0,3-0,7 segundos (s). El reflujo medio oscila entre 0,5 y $1,2 \mathrm{~s}$ y el grave entre 3 y 4 s (64). En la tabla III se recogen las diferencias ecográficas más características de las TVP agudas y crónicas.

\section{TABLA III}

\section{DIFERENCIAS ECO GRÁFICAS ENTRE LAS TROM BOSIS VENOSAS PRO FUNDAS AGUDAS Y CRÓNICAS}

\begin{tabular}{|c|c|c|}
\hline Características & Agudas & Crónicas \\
\hline Aspecto venoso & $\begin{array}{l}\text { Venas distendidas, } \\
\text { de paredes normales }\end{array}$ & $\begin{array}{l}\text { Venas de calibre normal, } \\
\text { paredes gruesas y ecoicas }\end{array}$ \\
\hline $\begin{array}{l}\text { Efecto de } \\
\text { la compresión }\end{array}$ & $\begin{array}{l}\text { Limitada o no } \\
\text { compresible }\end{array}$ & Parcialmente compresible \\
\hline Aspecto del trombo & Anecoico o ecoico & $\begin{array}{l}\text { Ecoico, paredes retraídas, } \\
\text { áreas parcheadas }\end{array}$ \\
\hline Doppler & $\begin{array}{l}\text { Limitado, ausencia } \\
\text { de flujo }\end{array}$ & $\begin{array}{l}\text { Flujo anterógrado, } \\
\text { reflujo }\end{array}$ \\
\hline
\end{tabular}

Tomado de Fraser and Anderson (62).

Con la incorporación de técnicas especiales, como las pulsaciones secuenciales en gradiente de eco, la angioresonancia magnética puede diferenciar una TVP aguda de una crónica (65), lo que es muy importante si tenemos en cuenta que el $15 \%$ de los pacientes tienen episodios repetidos de trombosis que pueden ser confundidos con un SPT y, en consecuencia, recibir un tratamiento incorrecto (66). Las imágenes obtenidas con esta técnica evidencian que la trombosis aguda tiende a llenar y/o a distender el vaso. El trombo, generalmente, tiene una señal de baja intensidad y es relativamente homogéneo. Con tratamiento y el paso del tiempo presenta un incremento en la intensidad de la señal y es menos voluminoso. El diámetro luminal disminuye y se acompaña de un flujo lento y un engrosamiento aparente de su pared (65). Además es un método sensible en la detección de los cambios experimentados por los tejidos blandos como el edema subcutáneo, la fibrosis, la fasciosclerosis y las transformaciones degenerativas musculares. La tomografía computarizada muestra de una manera más clara los cambios óseos y las calcificaciones (67).

\section{MÉTODOS PARA LA INVESTIGACIÓN DE LA MICROCIRCULACIÓN}

Los estudios de la microcirculación, actualmente, están indicados únicamente en pacientes seleccionados y con propósitos de investigación. Los principales son $(12,68)$ :

- La flujometría láser Doppler, utilizada para calcular el flujo sanguíneo cutáneo local en respuesta a distintos estímulos, como la oclusión arterial, el ortostatismo, el estrés térmico, o para evaluar los cambios espontáneos del flujo sanguíneo a lo largo del tiempo.

- La medición transcutánea de la presión parcial de $\mathrm{P}_{2}$ y $\mathrm{PCO}_{2}$, empleada para el estudio de la hipertensión venosa, en la que se compara la tensión de oxígeno cutáneo con las características morfológicas capilares. Los pacientes con cambios morfológicos en los capilares sanguíneos, pero sin una reducción de los mismos, presentan una disminución significativa de la $\mathrm{PCO}_{2}$.

- La pletismografía por impedancia para calcular los cambios de volumen que se producen en el compartimento extracapilar como consecuencia de la filtración capilar.

\section{TRATAMIENTO DEL SPT}

\section{TRATAMIENTO MÉDICO DEL SPT}

Los fármacos flebotrópicos son la estrategia terapeútica de elección para los pacientes con SPT en los que no está indicada la cirugía o en los que ésta es un coadyuvante del tratamiento médico (63). Son productos naturales, seminaturales o sintéticos y, algunos de ellos, combinan dos o más principios activos para mejorar su eficacia. La mayor parte pertenecen a la familia de los flavonoides. Sus mecanismos de acción varían, pero la propiedad principal de todos ellos es la activaciòn del retorno venoso y linfático (63). Clínicamente mejoran el edema, ya que actúan sobre la microcirculación al disminuir la permeabilidad endotelial, la liberación de sustancias inflamatorias, y la adhesión de los leucocitos a la vez que inhiben los radicales libres. También están indicados para mejorar los síntomas funcionales y subjetivos, como la fatiga, los calambres nocturnos, la pesadez de las piernas y la tensión $(63,69)$.

En los últimos diez años se ha hecho más evidente la relación macro-microcirculación en la mayor parte de los SPT graves; mucho antes ya estaba clara que la relación entre el reflujo y la hipertensión venosa era un factor importante en el daño capilar $(70,71)$. Muchas investigaciones básicas y algunos estudios en humanos han confirmado el efecto de algunos flebotrópicos, particularmente la diosmina-hesperidina, sobre 
la microcirculación deteriorada por el SPT $(72,73)$. El efecto de los enzimas fibrinolíticos, como la urocinasa, está documentado en varios trabajos (74,75). Los glicosaminoglicanos,- como el suloxide (76), el heparán sulfato (77) y el defibrotide (78), tienen actividad fibrinolítica.

Entre los vasodilatadores, el efecto de la pentoxifilina $(9,79)$ así como el de la prostaglandina E1 $(9,80)$ para el tratamiento de las úlceras está bien documentado. La única indicación de la aspirina, es el tratamiento coadyuvante para la curación de las úlceras, debido a su acción antiagregante plaquetaria $(9,81)$.

En los últimos años se ha recomendado el uso contínuo de las medias de compresión, al menos en los dos años siguientes al episodio trombótico agudo, con el fin de prevenir la aparición del SPT $(8,9,15)$. Sin embargo, a la luz de los conocimientos actuales y la experiencia clínica (11), los síntomas agudos -el dolor y el edema-, suelen resolverse espontáneamente dentro de los tres meses siguientes a la TVP aguda, simplemente con la elevación de las piernas o evitando las posiciones o las actividades que las agravan. Sus desventajas potenciales incluyen su alto costo -más de $100 €$ - la dificultad de algunos pacientes para colocárselas, el calor y la falta de confort sobretodo en los meses de verano. Su uso está contraindicado en la insuficiencia arterial, ya que la compresión realizada puede empeorar la claudicación. Por tanto, parece razonable esperar por lo menos un año dado que, generalmente, estos pacientes no desarrollan un empeoramiento sustancial de su sintomatología y prácticamente ninguno presenta secuelas importantes. Si al cabo de este tiempo no tienen los síntomas típicos del SPT, deben evitarse las medias de compresión, ya que el riesgo de padecerlo es muy bajo (11). Para aquellos en los que no sean efectivas, o no las toleren, se recomienda la compresión neumática intermitente que mejora la sintomatología clínica en el 75\% de los casos (82).

\section{TRATAMIENTO QUIRÚRGICO DE LA INSUFICIENCIA VENOSA PROFUNDA}

Los candidatos a la cirugía venosa profunda son todos aquellos pacientes que sufren de insuficiencia venosa crónica grave, con reflujo venoso significativo e hipertensión venosa ambulatoria (63). Generalmente, antes de plantearse una intervención para corregir la insuficiencia valvular del sistema venoso profundo, se ha recurrido, sin éxito, a la terapia conservadora, a la cirugía del sistema venoso superficial y a la de las perforantes insuficientes, aunque a menudo estas intervenciones ya se han realizado de manera sucesiva (63).

Cuando el reflujo venoso profundo es ligero, la ligadura de la vena safena puede proporcionar un considerable beneficio al paciente y erradicar el reflujo en la vena femoral (83). Sin embargo, si el reflujo es importante, el sistema venoso profundo requerirá cirugía directa, teniendo en cuenta el alto porcentaje de úlceras recurrentes después del tratamiento conservador y los excelentes y duraderos resultados obtenidos por los centros que han optado por este tipo de tratamiento (63).

La cirugía valvular reconstructora incluye métodos directos que ayudan a restaurar la competencia valvular y métodos indirectos que mejoran la hemodinámica venosa del miembro afectado. Los métodos quirúrgicos directos están indicados cuando las válvulas bicúspides están dilatadas o prolapsadas pero todavía funcionan. En el SPT o en la agenesia valvular, cuando las válvulas están dañadas o ausentes, una técnica indirecta, es la mejor elección terapéutica (63).

El SPT es una patología compleja en el que la circulación venosa afecta muchos niveles y sistemas. Una rápida curación de las úlceras de estasis puede obtenerse por tanto mediante la corrección de todos los puntos de reflujo y el mantenimiento o la mejoría de los canales de retorno venoso. Dependiendo de la localización y extensión de la lesión valvular pueden utilizarse una variedad de técnicas quirúrgicas.

En una revisión de 423 reconstrucciones Raju et al (84), enumeran la duración de los logros alcanzados por los distintos métodos quirúrgicos, monitorizados con eco-Doppler, en el siguiente orden:

-Valvuloplastia interna

- Valvuloplastia interna con manguito protésico

- Valvuloplastia externa con sutura directa

-Injerto venoso

No hay diferencias significativas en la recurrencia de las úlceras con los distintos métodos utilizados.

\section{LA ESCLEROTERAPIA}

Es la obliteración química de las venas varicosas. Sus principales indicaciones son (63):

- Telangiectasias. Su tratamiento es especialmente estético.

- - Varices con un diámetro entre 1-3 mm en ausencia de reflujo valvular detectable por Doppler duplex.

-Venas residuales postquirúrgicas inferiores a $3 \mathrm{~mm}$ de diámetro.

-Venas varicosas recurrentes postquirúrgicas, si se originan a partir de una vena perforante inferior a $4 \mathrm{~mm}$ de diámetro.

-Varices de malformaciones venosas (tipo Klippel-Trenaunay), en las que la cirugía no es aconsejable

- Como tratamiento de urgencia en las hemorragias secundarias a la rotura de las venas varicosas

-Venas varicosas periulcerosas. La escleroterapia favorece su curación, al eliminar temporalmente la hipertensión venosa presente en el área ulcerosa.

Las contraindicaciones absolutas incluyen la alergia a las soluciones esclerosantes, las enfermedades sistémicas descompensadas, la TVP reciente, las infecciones sistémicas o locales, los edemas de miembros inferiores, la inmovilización y la isquemia crítica de los miembros inferiores. Las contraindicaciones relativas incluyen los pacientes con historia de TVP recurrentes, con trombofilia confirmada, mujeres en tratamiento con estrógenos/progestágenos y embarazadas.

La compresión,- vendas o medias elásticas -, mejora los resultados de la escleroterapia.

\section{CONCLUSIONES}

El SPT está inseparablemente unido a la hipertensión venosa y es acompañado de daños y reestructuración de los tejidos vasculares, dérmicos y subcutáneos. Estas lesiones tisulares pueden iniciarse por la generación de una reacción inflamatoria. Los indicadores inflamatorios incluyen el aumento de la permeabilidad endotelial, la unión de los leucocitos circulantes al endotelio, la infiltración por monocitos, linfocitos y mastocitos del tejido conectivo, y el desarrollo de infiltrados tisulares fibróticos y distintos marcadores molecu- 
lares, como el factor de crecimiento o las moléculas de adhesión a las membranas celulares. Los indicadores de la reación inflamatoria son detectados en los estadios precoces del SPT y pueden estar implicados en el desarrollo de la disfunción valvular venosa primaria. Una de las más importantes cuestiones que quedan por dilucidar es la identificación de los mecanismos desencadenantes de la reacción inflamatoria en el SPT. Los datos actuales sugieren que, entre los muchos mecanismos posibles ( hipoxia, estimulación humoral), un cambio en la tensión de cizallamiento de los fluídos desde niveles fisiológicos normales y una distensión endotelial bajo la influencia de una presion venosa elevada podrían actuar como mecanismos desencadenantes de la inflamación.

Aproximadamente el $30 \%$ de los pacientes con un primer episodio de TVP desarrollarán manifestaciones postrombóti- cas. Esta incidencia es considerablemente más baja que las aportadas por los antiguos ensayos clínicos. La mayor parte de los casos de SPT aparecen en los dos años siguientes al episodio trombótico agudo y, aparentemente, no tienen relación con la localización ni la extensión inicial de la trombosis venosa. El desarrollo de una trombosis recurrente ipsilateral conlleva un alto riesgo de padecer un SPT, por otra parte raro en pacientes con una primera trombosis adecuadamente anticoagulada. Aunque su pronóstico es menos desfavorable de lo que se creía en el pasado, es una causa importante de incapacidad con una considerable carga económica tanto para el paciente como para los servicios nacionales de salud. La prevención de la trombosis recurrente y el uso apropiado de medias elásticas, posiblemente, pueda minimizar el riesgo de aparición de las complicaciones a largo plazo de las trombosis venosas.

\section{Bibliografía}

1. Kierkegaard A. Incidence of acute DVT in two districts. A phlebographic study. Acta Chir Scand 1980; 146: 267-9.

2. Anderson FA, Wheeler HB, Goldberg RJ, Hosmer DW, Patwardhan NA, Jovanovic B,et al. A population-based perspective on the hospital incidence and case-embolism. The Worcester DVT Study. Arch Intern Med 1991; 151: 933-938

3. Ascari E, Siragusa S, Piovela F. The epidemiology of deep vein thrombosis and pulmonary embolism. Haematologica 1995; 80 (suppl 2) : 36-41.

4. Prandoni P, Lensing A, Cogo A, Cuppini S, Villalta S, Carta M, et al. The long-term clinical course of acute deep venous thrombosis. Ann Intern Med 1996; 125: 1-7.

5. Johnson BF, Manzo RA, Bergelin RO, Strandness DE. The site of residual abnormalities in the leg veins in long-term follow-up after deep vein thrombosis and their relationship to the development of the postthrombotic syndrome. Int Angiol 1996; 15: 14-9.

6. Khan SR, Solymoss S, Lamping Dl, Abenhaim L. Long-term outcomes after deep vein thrombosis: Post-phlebitic syndrome and quality of life. J Gen Intern Med 2000; 15: 425-9.

7. Leizorovicz A. Long-term consequences of deep vein thrombosis. Haemostasis 1998; 28 (Suppl 13): 1-7.

8. Ziegler S, Schillinger M, Maca TH, Minar E. Post-thrombotic syndrome after primary event of deep venous thrombosis 10 to 20 years ago. Thromb Res 2001; 101: 23-33.

9. Bernardi E, Prandoni P. The post-thrombotic syndrome. Curr Opin in Pulm Med 2000; 6: 335-42.

10. Saarinen J, Kallio M, Letho S, Hiltunen S, Sisto T. The occurrence of the post-thrombotic changes after an acute deep venous thrombosis. J Cardiovasc Surg 2000; 41: 441-8.

11. Ginsberg JS, Hirsh J, Mmath JJ, Vander LaandVries M, Magier D, Mackinnon B, et al. Prevention and treatment of postphlebitic syndrome. Arch Intern Med 2001; 161: 2105-9.

12. Nicolaides AN. Investigation of chronic venous insufficiency. Circulation 2000; 102: 126-63.

13. Bauer G. Roentgenological and clinical study of the sequelae of thrombosis. Acta Chir Scand 1942; 86 (suppl 74): 1-10.

14. Holmström M, Aberg W, Lockner C, Paul C. Long term clinical followup in 256 patients with deep-vein thrombosis initially treated with either unfractionated heparin or dalteparin: a retrospective analysis. Thromb Haemost 1999; 82: 1222-6.

15. Heit JA, Silverstein MD, Mohr DN, Petterson TM, Lohse CM, O'fallon WM, et al. The Epidemiology of venous thromboembolism in the community.Thromb Haemost 2001; 86: 452-63.

16. Strandness DE, Langlois Y, Cramer M, Randlet A, Thiele BL. Longterm sequelae of acute venous thrombosis. JAMA 1983; 250: 1289-92.

17. Beyth RJ, Cohen AM, Landefeld CS. Long-term outcomes of deep-vein thrombosis. Arch Intern Med 1995; 155: 1031-7.

18. Brandjes DPM, Buller HR, Heijboer H. Randomised trial of effect of compression stockings in patients with symptomatic proximal-vein thrombosis. Lancet 1997; 349: 759-62.
19. Villalta S, Bogatella P, Piccioli A, Lensing AWA, Prins MH, Prandoni P. Assessment of validity and reproducibility of a clinical scale for the post-thrombotic syndrome. Haemostasis 1994; 24 (suppl 1) 158 a.

20. Rosendo A, Fernandez D, Lucio R, Latorre J. Epidemiología. In: Güell J, Rosendo A, editors. Enfermedad Tromboembólica venosa ( E.T.E.V.). Síndrome Postrombótico. Barcelona: EDIKA MED; 1995 p. 1-5..

21. Bonal de Falgas J. Comité de profilaxis de la enfermedad tromboembólica. Estudio económico de la E.T.E.V Incidencia de T.V.P. y costos de los tratamientos. Boletín del comité de profilaxis de la enfermedad tromboembólica 1992; 4: 3-25.

22. Ruckley CV. Socioeconomic impact of chronic venous insufficiency and legs ulcers. Angiology 1997; 48: 67-69.

23. Schmid- Schönbein GW, Takase S, Bergan JJ. New advances in the understanding of the pathophysiology of chronic venous insufficiency . Angiology 2001; 52: S 27- S 28 .

24. Khan SR, Ginsberg JS. The post-thrombotic syndrome: current knowledge, controversies, and directions for future research. Blood Rev 2002; 16: $155-165$.

25. Burnand KG, Whinster I, Naidoo A, Browse NL. Pericapillary fibrin in the ulcer-bearing skin of the leg: The cause of lipodermatosclerosis and venous ulceration. BMJ 1982; 285: 1071-2

26. Pappas PJ, Gwertzman GA, Defouw DO, Padberg FT Jr, Silva MBR Jr, Duran WN, et al. Retinoblastoma protein: A molecular regulator of chronic venous insufficiency. J Surg Res 1998; 76: 149-53.

27. Weyl A, Vanscheit W, Weiss JM, Peschen M, Schopf E, Vestweber D, et al. Expression of the adhesion molecules ICAM-1, VCAM-1, and Eselectin and their ligands VLA-4 and LFA-1 in chronic venous leg ulcers. J Am Acad Dermatol 1996; 34: 418-23.

28. Semenza GL, Wang GL. A nuclear factor induced by hypoxia via de novo protein synthesis binds to the human erythropoietin gene enhacer at a site required for transcriptional activation. Mol Cell Biol 1992; 12: 5447-54.

29. Haidekker MA, L'heureux N, Frangos JA. Fluid shear stress increases membrane fluidity in endothelial cells: A study with DCVJ fluorescence. Am J Physiol Heart Circ Physiol 2000; 278: H 1401-H 1406.

30. Gudi S, Nolan JP, Frangos JA. Modulation of GTPase activity of G proteins by fluid shear stress and phospholipid composition Proc Natl Acad Sci USA 1998; 95: 2515-9.

31. Arnoldi CC. Venous pressure in patients with valvular incompetence of this veins of the lower extremities. Acta Chir Scand 1966; 132: 628-33.

32. Bao X, Clark CB, Frangos JA. Temporal gradient in shear-induced signalling pathway: Involvement of MAP kinase, C-fos, and connexin 43. Am J Physiol Hearth Circ Physiol 2000; 278: H 1598- H 1600.

33. Resnick N, Yahav H, Khachigian LM, Collins T, Anderson KR, Dewey FC, et al. Endothelial gene regulation by laminar shear stress. Adv Exp Med Biol 1997; 430: 155-64.

34. Thomas PRS, Nash GB, Dormandy JA. White cell accumulation in dependent legs of patients with venous insufficiency: A possible mechanism for trophic changes in the skin. BMJ 1988; 296: 1693-169. 
35. Saharay M, Shields DA, Porter JB, Scurr JH, Coleridge S. Leukocyte activity in the microcirculation of the leg in patients with chronic venous disease. J Vasc Surg 1997; 26: 265-73.

36. Burnand KG, Clemenson G, Whimster I, Gaunt J, Browse NL. The effect of sustained venous hypertension on the skin capillaries of the canine hind limb. Br J Surg 1982; 69: 41-4.

37. Moazam F, Delano FA, Zweifach BW. The leukocyte response to fluid stress. Proc Natl Acad Sci USA 1997; 94: 5338-43.

38. Fukuda S, Yasu T, Predescu DN, Scmid-Schönbein GW. Mechanism for regulation of fluid shear stress response in circulating leukocytes. Cir Res 2000; 86: E 13-E 18.

39. Hynes RO. Integrins: Versatility, modulation, and signaling in cell adhesion. Cell 1992; 69: 11-25.

40. Wenner A, Leu HJ, Spycher M. Ultrastructural changes of capillaries in chronic venous insufficiency. Expl Cell Biol 1980; 48: 1-4.

41. Takase S, Lerond J, Bergan JJ, Schmid-Schönbein GW. The inflammatory reaction during venous hypertension in the rat. Microcirculation 2000; 7: 1-11.

42. Suematsu M, Delano FA, Poole D, Engler RL, Miyaska M, Zweifach BW, et al. Spatial and temporal correlation between leukocyte behavior and cell injury in postischemic rat skeletal muscle microcirculation. Lab Invest 1994; 70: 684-95.

43. Labropoulos N, Giannoukas AD, Delis K, Mansour MA, Kang SS, Nicolaides NN, et al. Where does venous reflux start? J Vasc Surg 1997; 26: 736-42.

44. Davenpeck KL, Gauthier TW, Lefer AM. Inhibition of endothelial-derived nitic oxide promotes P-selectin expression and actions in the rat microcirculation. Gastroenteroloy 1994; 107: 1050-1058.

45. Harris AG, Costa JJ, Delano FA, Zweifach BW, Schmid-Schönbein GW. Mechanisms of cell injury in rat mesentery and cremaster muscle. Am J Physiol 1998; 274: H 1009-15.

46. Mori N, Horie Y, Gerritsen ME, Granger DN. Ischemia-reperfusion induced microvascular responses in LDL- receptor -/- mice. Am J Physiol 1999; 276: H 1647- H 1654.

47. Lalka SG, Unthank JL, Nixon JC. Elevated cutaneous leukocyte concentration in a rodent model of acute venous hypertension. J Surg Res 1998; 74: 59-63.

48. Mashiah A, Berman V, Thole HH, Rose SS, Pasik S, Schwarz H, et al. Estrogen and progesterone receptors in normal and varicose saphenous veins. Cardiovasc Surg 1999; 7: 327-331.

49. Pitzer JE, Del Zoppo GJ, Schmid-Schönbein GW. Neutrophil activation in smokers. Biorheology 1996; 33: 45-58.

50. Browse NL, Clemenson G, Lea TM. Is the postphlebitic leg always postphlebitic? Relation between phlebographic appearances of deepvein thrombosis and late sequelae. BMJ 1980; 281: 1167-70.

51. Lindner DJ, Edwards JM, Phiney ES,Taylor LM Jr, Porter JM. Longterm hemodynamic and clinical sequelae of lower extremity deep vein thrombosis. J Vasc Surg 1986; 4: 436-42.

52. Villanueva EL, Anderson JN, Gan E T. Does the location of deep venous thrombosis affect the risk of developing postphlebitic syndrome? Med J Aust 2001; 174: 101-2.

53. Trubestein G. Can thrombolytics prevent post-phlebitic syndrome and thromboembolic disease? Hemostasis 1986; 3 (suppl 6): 38-50.

54. Mewissen MW, Seabrook GR, Meissner MH, Cynamon J, Labropoulos $\mathrm{N}$, Haughton SH. Catheter-directed thrombolysis for lower extremity deep venous thrombosis: report of a national multicenter registry. Radiology 1999; 211: 39-49.

55. Palareti G, Legnani C, Cosmi B, Guazzaloca G, Pancani C, Coccheri S. Risk of venous thromboembolism recurrence: High negative predictive value of D-dimer performed after oral anticoagulation is stopped. Thromb Haemost 2002; 87: 7-12.

56. Biguzzi E, Mozzi E, Alatri A, Taioli E, Moia M, Mannucci PM. The post-thrombotic syndrome in youn women: Retrospective evaluation of prognostic factors. Thromb Haemost 1998; 80: 575-7.

57. Kearon C, Gent M, Hirsh J, Weitz J, Kovacks MJ, Anderson DR, et al. A comparison of three months of anticoagulantion with extended anticoagualation for first episode of idiopathic venous thromboembolism. N Engl J Med 1999; 340: 901-7.

58. Agnelli G, Prandoni P, Santamaria MG, Bagatella P, Iorio A, Bazzan M, et al. Three months versus one year of oral anticoagulant therapy for idiopathic deep venous thrombosis. N Engl J Med 2001; 345: 165-9.
59. Torres R. Clínica y diagnóstico diferencial de la trombosis venosa profunda y del síndrome postrombótico. In: Güell J, Rosendo A, editors. Enfermedad Tromboembólica venosa ( E.T.E.V.). Síndrome Postrombótico. Barcelona: EDIKA MED; 1995, p. 21-46.

60. Rutherford RB, Padberg FT, Comerota AJ, Kistner RL, Meissner MH, Moneta GL. Venous severity scoring: An adjunct to venous outcome assessment. J Vasc Surg 2000; 31: 1307-1312.

61. Kamida CB, Kistner R, Elkof B, Masuda EM. Lower extremity ascending and descending flebography. En : Gloviczki P, Yao JST. eds. Handbook of venous disorders 2nd Edition. NY: Arnold, 2001; 132-9.

62. Fraser JD, Anderson DR. Deep venous thrombosis: Recent advances and optimal investigations with US. Radiology 1999; 211: 9-24.

63. Agus GB, Allegra C, Arpaia G, Botta G, Cattaldi A, Gasbarro V, et al. Guidelines for the diagnosis and treatment or chronic venous insufficiency. Int Angiol 2001 ; 20: 3-37.

64. Mattos MA, Sumner D. Direct noninvasive tests (duplex scan ) for the evaluation of chronic venous obstruction and valvular incompetence. In:Gloviczki P, Yao JST. editors. Handbook of venous disorders 2 nd ed. NY: Arnold; 2001, p. 120-131.

65. Spritzer CE, Trotter P, Sostman HD. Deep venous thrombosis: gradientrecalled-echo MR imaging changes over time-experience in 10 patients. Radiology 1998; 208: 631-639.

66. Dorfman GS, Cronan JJ. Asymptomatic pulmonary embolism in deep venous thrombosis: long-term follow-up. Radiology 1987: 165: 205211.

67. List- Hellwig E, Meents H. Magnetic resonance imaging and computed tomography in advanced chronic venous insufficiency. Current Probl Dermatol 1999; 27: 109-13.

68. Cesarone MR, De Sanctis MT, Incadela L, Belcaro G, Griffin M, Cacchio M. Methods of evaluation an quantification of microangiopathy in high perfusion (chronic venous insufficiency an diabetic microangiopathy). Angiology 2001; 52 ( suppl 2): S 3-S 7.

69. Coleridge PD. Update on chronic-venous -insufficiency-induced inflamatoru processes. Angiology 2001; 52 (Suppl 1): S 35-S 42.

70. Wenner A, Leu HJ, Spycher M, Brunner U. Ultrastructural changes of capillaries in chronic venous insufficiency. Exp Cell Biol 1980; 48: 1-14.

71. Fagrell B. Microcirculatory disturbances: the final cause for venous leg ulcers? Vasa 1982; 11: 101-3.

72. Allegra C, Bartolo M, Carioti B, Cassiani D. An original microhaemorheological approach to the pharmacological effects of Daflon 500mg in severe chronic venous insufficiency. Int $\mathrm{J}$ Microcirc Clin and Exper 1995; 15 (suppl 1): 50-4.

73. Bergan JJ, Scmid-Schönbein GW, Takase S. Therapeutic approach to chronic venous insufficiency and its complications: place of Daflon ${ }^{\circledR}$ $500 \mathrm{mg}$. Angiology 2001; 52: S 43-S 47.

74. Ehrly AM, Schenk J, Bromberger U. Mikrozirkulationsstörungen bei ulcus cruris venosum: niedrigdosierte systemische Langzeit-therapie mit Urokinase. Phlebol Proktol 1989; 18: 166-8.

75. Partsch $\mathrm{H}$. Treatment of resistant leg ulcers by retrograde intravenous pressure infusions of urokinase. Phlebology 1991; 6: 13-21.

76. Harenberg J. Review of pharmacodynamics, pharmacokinetics, and therapeutic properties of sulodexide. Med Res Rev 1998; 18: 1-20.

77. Allegra C. Ruolo attuale dei glicosaminoglicani e prospettive in terapia. Min Angiol 1993; 18 (suppl 3) 45-9.

78. Cesarone MR, Belcaro G, Geroulakis G. Activation fibrinolytique par le defibritide dans le traitment des ulcères veineux. Angiologie 1997; 49: $10-14$

79. Weitgasser H. The use of pentoxifylline (Trental 400) in the treatment of leg ulcers: results of a doubled-blind trial. Pharmatherapeutica 1983; 3 (suppl 1): 143-51.

80. Rudofsky G. Intravenous prostaglandin E 1 in the treatment of venous ulcers - a double - blind, placebo - controlled trial. Vasa 1989; 28: 39-43.

81. Layton AM, Ibbotson SH, Davies JA, Goodfield MJ. Randomised trial of aspirin for chronic venous leg ulcers. Lancet 1994; 344: 164-5.

82. Ginsberg JS, Magier D, McKinnon B, Hirsh J. Intermittent compression units for severe postphlebitic syndrome: a randomized crossover study. VMAJ 1999; 160: 1303-6.

83. Walsh JC, Bergan JJ, Beaman S. Femoral venous reflux abolished by greater saphenous stripping. Ann Vasc Surg 1994; 8: 566-70.

84. Raju S, Neglèn P, Doodlite J, Meydrech EF. Axillary vein transfer in trabeculated post thrombotic veins. J Vasc Surg 1999; 29: 1050-64. 
